\title{
A Delay Recruitment Model of the Cardiovascular Control System
}

\author{
A.C. Fowler* M.J. McGuinness ${ }^{\dagger}$ \\ Accepted for publication in The Journal of Mathematical Biology, \\ April 2005. Copyright will be owned by Springer.
}

\begin{abstract}
We develop a nonlinear delay-differential equation for the human cardiovascular control system, and use it to explore blood pressure and heart rate variability under short-term baroreflex control. The model incorporates an intrinsically stable heart rate in the absence of nervous control, and allows us to compare the baroreflex influence on heart rate and peripheral resistance. Analytical simplifications of the model allow a general investigation of the rôles played by gain and delay, and the effects of ageing.
\end{abstract}

\section{Introduction}

Control of blood pressure is critical to human health. Hypertension (high blood pressure) is associated with increased risk of myocardial infarction, stroke, kidney failure and congestive heart failure. Elevated sympathetic tone (the mean level of activity of the sympathetic nervous system) is in turn linked with hypertension [16], and there is much interest in non-invasive diagnostic tools for assessing sympathetic tone and the baroreflex mechanisms that generate it. Any such assessment of baroreflex health, using natural variations in heart rate and blood pressure data, requires a deep understanding of the way that the controlled cardiovascular system behaves in the short term.

\footnotetext{
*Mathematical Institute, 24-29 St. Giles', University of Oxford, OX1 3LB, England

${ }^{\dagger}$ Division of Applied Mathematics, Korea Advanced Institute of Science and Technology, Taejon, South Korea, present address: School of Mathematical and Computing Sciences, Victoria University of Wellington, P.O. Box 600, Wellington, New Zealand
} 
Recently, the clinical physiology of cardiac control has become better understood and quantified, particularly under open-loop conditions (where parts of the control system are studied in isolation), leading to a number of mathematical models of this short-term pressure control system. The challenge for these models is to explain and understand the rôles played by different components of the system. Nonlinear feedback loops with different delays complicate the response of the system to changes. The bio-rhythms associated with baroreflex control remain the subject of intensive study and debate as to their specific origin. Understanding the way that natural variability in blood pressure and heart rate depend on the health of the various parts of the control system is central to non-invasive clinical techniques, particularly those that seek to assess sympathetic tone.

We discuss the bio-rhythms associated with short-term variability in the human cardiovascular system in the next section. Then we present the elements of physiology needed to inform a mathematical model of the cardiac control system. Selected existing mathematical models are then briefly reviewed. Then, a simple new model for blood pressure and heart rate is presented, incorporating both neural and mechanical feedback mechanisms.

This model can be reduced to a single delay recruitment type equation. This simplification of the model is significant, in that it allows a deeper, more analytical and more general mathematical analysis than is usually possible in cardiovascular models. The three feedback loops operating in the model have inherently complicated consequences for solution behaviour, but our model reduction allows us to simplify these effects while retaining the key features of nonlinearity and time delay.

Steady and periodic solutions are studied with analytical tools, in a detailed exploration of how the behaviour of the system depends on sympathetic feedback mediated by the baroreflex mechanism. In particular, the rôles of delay times and feedback amplitudes are explored and discussed, together with the consequences of ageing.

\subsection{Rhythms observed in human cardiac systems}

The earliest and most well-known rhythmic variation associated with blood pressure is respiratory sinus arrhythmia (RSA), in which heart rate increases during inspiration. Two important parts of the automatic central nervous system for controlling the heart are the sympathetic and the parasympathic (or vagal) nervous systems. Roughly speaking, the sympathetic system is responsible for preparing us to fight or run, and the vagal system acts re-

ciprocally, as well as controlling the viscera. The vagal nervous system is 
known to participate in RSA, both through a central coupling with respiration controls and through the baroreflex $[26,2]$. The period of this cyclic oscillation in blood pressure and heart rate matches respiration, at about 3-6 seconds. Since we are here particularly interested in sympathetic tone rather than vagal influences, we do not model RSA in this paper, but regard it as a source of perturbation of blood pressure.

Slower oscillations of blood pressure, with a period of about 10 seconds, are termed Mayer waves. There has been some debate about their cause [26]. An oscillator of the requisite frequency in the central nervous system has been suggested $[10,27,14]$, but the most commonly ascribed cause is sympathetic (delayed) feedback control of blood pressure through the baroreflex control system [11, 5, 6, 23, 34, 36].

There remains some disagreement over whether Mayer waves are due to (and most sensitive to) the gain in the baroreceptors [1], or the delay in their feedback $[6,34]$. There has also been evidence presented that the sympathetic control of peripheral resistance is more important than sympathetic feedback to the heart muscle or heart rate changes [23, 26, 31].

These are the most important types of short-term variability in the cardiovascular system. We do not consider longer-term effects such as blood chemistry, renal moderation of blood volume, or thermal and hormonal responses.

\section{Physiology}

An excellent discussion of modelling the physiology of the human cardiovascular control system is presented by Ottesen [29, 30]. We here present a summary of the physiology, to motivate the model to be developed in section (4). Our discussion is necessarily much simplified, and the reality of cardiovascular action and control is much more complicated [18]. The key elements in the short-term control of the human cardiac system are illustrated in Fig. (1).

The heart pumps blood around the body (the systemic loop), and through the lungs (the pulmonary loop). The action is pulsatile, with a typical resting heart rate of about 70 beats per minute. Blood ejected from the left ventricle enters the arteries at a relatively high pressure. The arteries are compliant (can stretch elastically) and pass the blood on to the smaller arterioles and capillaries, where most of the resistance to flow resides. Blood then enters the compliant veins at a relatively low pressure, and passes back to the heart. The veins have a relatively small resistance to flow, and the 


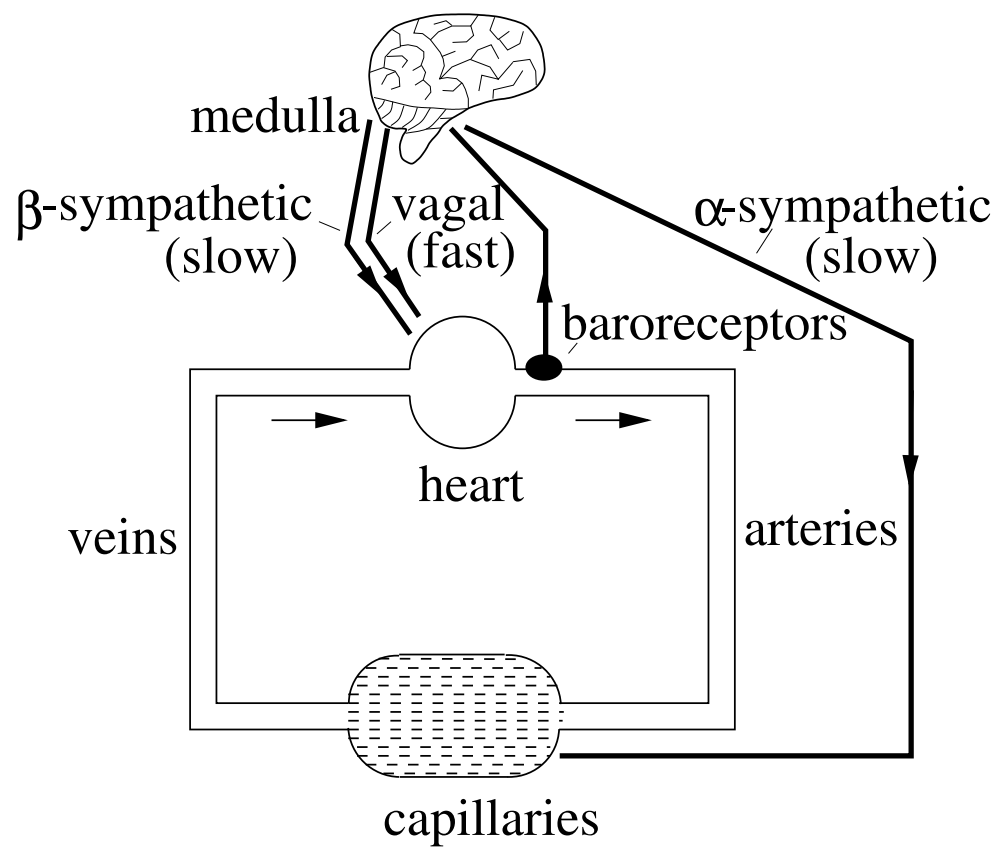

Figure 1: A sketch of the short-term baroreflex cardiac control system. 
flow is less pulsatile there.

Starling's Law of the heart (e.g. [33], p.175) says the strength of a contraction increases with increasing myocardial fibre length, so that a longer filling time gives a stronger contraction.

The relationship between the highest value of blood pressure (systolic, just as the heart contracts) and the lowest value of blood pressure (diastolic, just before the next contraction) may be related to the effective resistance and capacitance of the blood vessel system by a Windkessel model which describes the exponential decay of arterial pressure with time between heart beats. This model is essentially a conservation of mass model.

The heart contraction that pumps blood to the arteries is usually initiated in the sino-atrial node, which is located in the heart wall. This node can initiate a contraction spontaneously, and will do so in the absence of nervous control, pulsing at about 100 beats per minute. But the action of the sino-atrial node is controlled by electrical pulses sent along nerves from the medulla to the heart. There are two major systems of these nerves, the sympathetic and the parasympathetic systems, which act together in a double-action control mechanism.

The vagal or parasympathetic nervous system is relatively fast-acting. Heart rate is reduced within a time less than the time between heart beats, when extra signals arrive along the vagus nerve via a fast-acting (and rapidly degradable) chemical called acetylcholine. Conversely, the heart rate increases in response to sympathetic signals, via the much slower chemical action of norepinephrine. There can be delays of $2-5$ seconds before sympathetic changes take effect. Both nervous systems affect the heart - if vagal activity changes, the heart rate will respond. If sympathetic activity changes, the heart rate will respond, although more slowly. Usually, both systems change together, with an increase in vagal activity accompanied by a decrease in sympathetic activity, for example. Furthermore, there is some evidence that the vagus can pre-empt sympathetic activity.

These nervous signals also have other short term effects on the heart, including altering the contractility and as a consequence the stroke volume. Sympathetic signals also reach the capillaries and arterioles, increasing their resistance to flow when the signal rate (tone) increases.

Baroreceptors are nerve fibre endings in arterial walls, with key receptors in the aortic arch and in the carotid sinus artery in the neck. They are sensitive to the average arterial blood pressure, and to the rate at which blood pressure increases, firing more often during the time when the pulsatile pressure is increasing.

When blood pressure rises, for example, the baroreceptors fire more sig- 
nals to the medulla in the brain, which usually responds by sending more signals along the vagal system, and fewer signals along the sympathetic system. The combined effect of this double control system is to reduce heart rate, which in turn allows the arterial blood pressure to drop, correcting the perceived change. The baroreceptor response to arterial pressure is nonlinear [21], and is attenuated at very high and very low pressures.

There are strong clinical links between sympathetic activity and Mayer waves - if sympathetic activity is chemically blocked, Mayer waves are significantly reduced or completely eliminated [4, 15, 3, 8]. Indeed, Guyton and Harris [19] proposed the term vasomotor waves for blood pressure oscillations slower than respiration. Vasomotor refers to the smooth muscle in vascular walls, whose behaviour is controlled by the sympathetic nervous system, but which is also known to vary spontaneously.

\section{Existing Models}

A number of approaches have been taken to modelling and understanding the short-term control of blood pressure. Challenges include the nonlinearity of the baroreflex and medulla responses, the various time delays, and the number of different feedback loops. Another consideration for modelling is whether to explicitly include the pulsatile nature of blood flow, or simply to consider an average pressure.

An early modelling approach was Grodins' [17] system of algebraic equations for the steady controlled heart, which is rearranged to give a sixth degree polynomial for mean arterial pressure. A closed mechanical heart system is controlled directly by the central nervous system and by the action of endocrines. Starling's Law and a Windkessel model are used, and the parameters are fitted to available experimental results. The effects of changing peripheral resistance, blood volume, heart rate and ventricular contraction strength are explored.

A number of modellers have used ordinary differential equations with delays, which is an attractive approach as it allows the model to be quite faithful to the physiology, and it offers some hope of analysing how oscillations in blood pressure depend on the various parameters, and relating this back to the physiology. Some of these differential (and difference) equation models are reviewed briefly here.

A seminal beat-to-beat difference model due to de Boer et al. [11] considers the discrete beating action of the heart. Each heart beat is considered as a discrete event, and the sympathetic feedback from the baroreceptors is 
distributed in a delayed manner over the following 2-6 heart beats. Sympathetic response to pressure is taken to be an arctan function, to give the characteristic sigmoidal response curve seen in clinical studies. The model incorporates baroreflex feedback to heart rate and peripheral resistance, Starling's Law, a Windkessel model of the systemic loop, and respiration effects on blood pressure, and is solved numerically. Oscillations similar to Mayer waves are observed. The model results showed that there are difficulties interpreting power spectra for estimating baroreflex gain, as it is frequency dependent.

Another difference equation model [32] considers central nervous control of heart rate with delay, using a one-third power or a hyperbolic tangent in two different approaches to approximating the sigmoidal baroreceptor response curve. The heart model is electric or nervous, allowing for recovery time after a contraction. The Windkessel heart model is not used, and the mechanical coupling of outflow through the arteries to inflow from veins is not explicitly included. The resulting four coupled difference equations are solved numerically with white noise forcing, and power spectra presented. Healthy and pathological spectra are observed, and bifurcation to low-dimensional chaos. A healthy chaotic spectrum computed from the numerical solution shows a peak near $0.1 \mathrm{~Hz}$, suggestive of Mayer waves.

The work of Madwed et al [24] is more descriptive than mathematical, and uses feedback control modelling. An equation-free model is set up, using low-pass filters in series with feedback delay. Detailed quantitative physiological information is provided that is useful for any mathematical model. The processes and timescales of baroreceptor feedback on HR and $\mathrm{BP}$ are discussed in detail. In particular, the delay between stimulation of arterial baroreceptors and the onset of afferent nerve activity is noted to be about 100-300 ms. This is the time required for the signals to travel from baroreceptors to the medulla, then to be processed by the medulla. It is also typical of the total times for vagal responses to be seen - the delay time between a change in arterial pressure and an observable vagally-mediated response at the heart.

Sympathetic nervous responses are much slower, and are more widely distributed in time. Typically there is a $2.5 \mathrm{~s}$ delay before $\beta$-sympathetic nerves begin to affect heart rate, and a further $7.5 \mathrm{~s}$ before that effect is complete (in the absence of any other changes). There is a $5 \mathrm{~s}$ delay before peripheral vasoconstriction begins, in response to stimulation of $\alpha$-sympathetic nerves, and a further $15 \mathrm{~s}$ delay before vasoconstriction is complete.

A number of differential-delay equation models have been developed in recent years. Ursino et al [37] consider the rôle of the venous capacity, in a 
detailed nonpulsatile two-compartment heart model using compliances and resistances for the systemic and pulmonary circulations, and with baroreceptor control of peripheral resistance, venous volume, and heart rate. They use exponentials to form the sigmoidal baroreceptor response curve, and solve numerically the resulting fourteen coupled ordinary differential equations with delays. Their model agrees well with experiment, and indicates that sympathetic control of venous capacity can smoothly compensate for a sudden loss of blood.

This model was extended subsequently [38] to allow for cardiac pulsatility, and more accurate baroreceptor modelling. Sigmoidal feedback with different lag times from baroreceptors to peripheral resistance, heart rate and venous capacity was included. There is a rate-dependent component in the baroreceptor response to pressure change. The model was presented as thirteen coupled nonlinear differential-delay equations, and was solved numerically using a control-theory approach (that is, by first linearising the equations). Open-loop (no pressure feedback to the baroreceptors) experiments were closely matched by the model simulations. The effects of including pulsatility were found to depend on mean arterial pressure levels. If these levels were low, pulsatility led to a reduced baroreflex. If they were high, pulsatility had the opposite effect. Stable pressures were observed for time delays of 2-5 seconds, with self-sustaining Mayer waves observed for time delays of more than $8-11$ seconds. It was noted that the onset and disappearance of these oscillations depended on the location of the steadystate (working point) about which the system was linearised, and on pressure pulse amplitude, in a complicated way.

This model was further modified [36] to include eighteen coupled nonlinear delay-differential equations, solved with a fully nonlinear numerical package. Good agreement with experimental results is obtained. Venous unstressed volume control is found to play the major rôle in early responses to haemorrhage.

A simpler model is presented by Ottesen [29], with no pulmonary circulation, and a single-compartment heart model, leading to two coupled differential equations for mean arterial and venous pressures. The baroreceptor response curves are kept general, and are taken to control heart rate only. Existence and uniqueness of an equilibrium are established, and its stability analysed under rather general conditions. Three coupled linear first-order delay-differential equations describing stability are obtained and studied. Oscillations consistent with Mayer waves are seen, and are sensitive to parameter values (delay, peripheral resistance, etc). The model response to a sudden change in peripheral resistance is consistent with experiment. 
A hybrid model, using an integrate-and-fire model of the sinus node to generate a discrete heart rate, but continuous variables otherwise, has been presented by Seidel and Herzel [34]. Baroreceptor response curves are sigmoidal, with sympathetic delays up to $5 \mathrm{~s}$ considered, and baroreceptor feedback is to the heart rate, peripheral resistance and cardiac contractility. The integrate-and-fire model allows more detailed and accurate modelling of the varying sensitivity of the sinus node to central control. Four differential equations, for a single-compartment Windkessel model of the heart driving just the systemic circulation, are solved numerically. Steady solutions bifurcate to periodic solutions, as time delays are increased. Under normal conditions, damped oscillations at Mayer wave frequencies are seen. Period-doubling bifurcations to chaos, and toroidal oscillations, are noted to occur provided the phase sensitivity of the sinus node is included. Return maps for sinus node response to single stimuli are generated, due to the integrate-and-fire feature. These compare well with experiment.

A more recent model [1] is able to produce Mayer waves without using any delays, simply by increasing the baroreceptor feedback gain. The model has a two-chamber heart, with both pulmonary and systemic circulations. Hill functions are used to give the sigmoidal baroreceptor response curves, and (instantaneous) feedback to heart rate, peripheral resistance, venous unstressed volume and venous compliance are each considered separately. Steady state solutions are obtained for the resulting three coupled nonlinear first-order differential equations, and the stability of the steady states is investigated. Hopf bifurcations to oscillatory solutions consistent with Mayer waves are obtained, but only in the cases of feedback to unstressed venous volume or venous compliance, and only with very large gains. Since the unstressed venous volume term appears with a relatively large time-constant in their differential equation for arterial volume/pressure, this is suggestive that changes in unstressed venous volume have effectively a delayed effect on arterial pressure.

Further modifications have been made [25] to Ursino's model [36], by adding pulmonary baroreceptors, respiratory influences, and a simplified baroreceptor feedback model. Delay differential equations are presented, using the language of control theory, and parameter values are assigned based on a careful analysis of the physiology. Numerical solutions are then used to explore response to acute haemorrhage - increased RSA is seen. A sensitivity analysis is also undertaken - the system is most sensitive to vagal gain and delay, less sensitive to the arterial baroreflex, and least sensitive to the pulmonary baroreflex. The system is inherently rather stable. 


\section{A simple model}

Our aim is to choose a model that is simple enough to allow substantial mathematical analysis, supported only peripherally by numerical computations, yet complex enough to be faithful to as much of the physiology as possible. We use nonlinear delay differential equations, for averaged cardiac variables. We explore the implications of this model for the sensitivity of Mayer waves to sympathetic delay and gain, to sympathetic control of peripheral resistance and of heart rate, and to the effects of ageing.

We follow Ottesen [29] and choose a nonpulsatile lumped-parameter model of the systemic loop, consisting of the left ventricle, which pumps blood to the arteries, the capillaries (and arterioles), the veins, and back to the left ventricle (via the right ventricle and the lungs). The pulmonary system is neglected here. Including it (e.g., as in [1]) would lead to one extra differential equation for the pulmonary venous pressure. Neglecting transient behaviour in the pulmonary system allows us to lump the action of the heart and the pulmonary system together into one cardiac output term. Besides simplifying the model, this reflects our more central interest in the systemic behaviour in this paper.

We further assume the system is closed with the blood being incompressibile so that blood volume is conserved, that large arteries and veins act like compliant vessels (so that volume changes are proportional to pressure changes), and that the capillary system is like a resistance vessel (with flow rate depending on the pressure drop across the system).

To this closed mechanical system we add the baroreflex control system (Fig. 1). The baroreflex sensors detect arterial pressure and send signals to the brainstem or medulla, which in response sends fast-acting (vagal, or parasympathetic) and slow-acting (sympathetic) signals back to the heart, altering heart rate, and to the arterioles and capillaries, altering the peripheral resistance. We neglect the small delay in the parasympathetic system, and we approximate the distributed delay of the sympathetic system with a single delay time.

Our model is essentially an extension of that introduced by Ottesen [29]. We have added an intrinsically controlled heart rate, and baroreflex control of peripheral resistance. In the absence of feedback from the central nervous system, the heart is known to continue to beat spontaneously at a rate set by the firing of the sino-atrial node. Our model explicitly includes this intrinsic controlled behaviour. We anticipate (and find) that this natural frequency has a significant effect on the dynamics of the response of the cardiac system to baroreflex feedback. The importance of peripheral resistance has been 
raised in a number of previous studies [23, 26, 29, 31], so it is useful here to also consider the relative importance of sympathetic control of peripheral resistance and sympathetic control of heart rate.

Then equations for the conservation of average (non-pulsatile) blood volume are:

$$
\begin{aligned}
C_{a} \dot{p}_{a} & =-\frac{\left(p_{a}-p_{v}\right)}{R_{c}}+H \Delta V \\
C_{v} \dot{p}_{v} & =\frac{\left(p_{a}-p_{v}\right)}{R_{c}}-\frac{p_{v}}{R_{v}} .
\end{aligned}
$$

Here, $p_{a}$ is the mean arterial pressure, $\dot{p}_{a}$ is its time rate of change, $p_{v}$ is the mean venous pressure, $C_{a}$ is the compliance of the arterial system, $C_{v}$ is the compliance of the venous system, $R_{c}$ is the resistance to flow through the arterial system, $R_{v}$ is the resistance to flow through the venous system, $H$ is heart rate and $\Delta V$ is the stroke volume (the volume pumped out in one heart beat).

Central nervous control in this model affects both the heart rate $H$ and the peripheral resistance $R_{c}$ :

$$
\dot{H}=\frac{\beta_{H} T_{s}}{1+\gamma T_{p}}-V_{H} T_{p}+\delta_{H}\left(H_{0}-H\right)
$$

and

$$
R_{c}=R_{c}^{0}\left(1+\alpha T_{s}\right)
$$

where $\beta_{H}$ is the strength of the sympathetic tone $T_{s}$ and $V_{H}$ is the strength of the parasympathetic or vagal tone $T_{p}$, both of which depend on the mean arterial pressure $p_{a}$. The sympathetic feedback $T_{s}$ is significantly delayed, by up to ten times the delay in parasympathetic feedback.

The parameter $\gamma$ is a measure of the direct damping effect of vagal activity on the sympathetic tone. Ottesen sets $\gamma=0$, reflecting the fact that even if $\gamma$ is of order one it has little effect on the steady heart rate dependence on $p_{a}$, it simply shifts the sigmoidal plot of steady $H$ vs. $p_{a}$ to the left a little. We use the nominal value $\gamma=0.2$.

The final term in the heart rate equation causes the heart (in the absence of central nervous control) to relax to the rate $H_{0}$ seen in a denervated heart, about 100 beats per minute. It is a simple representation of the idea that the oscillations of the sino-atrial pacemaker action potential are those of a limit cycle oscillator, and as such, it can be expected that both amplitude and phase (and thus heart rate) respond stably to perturbation. 


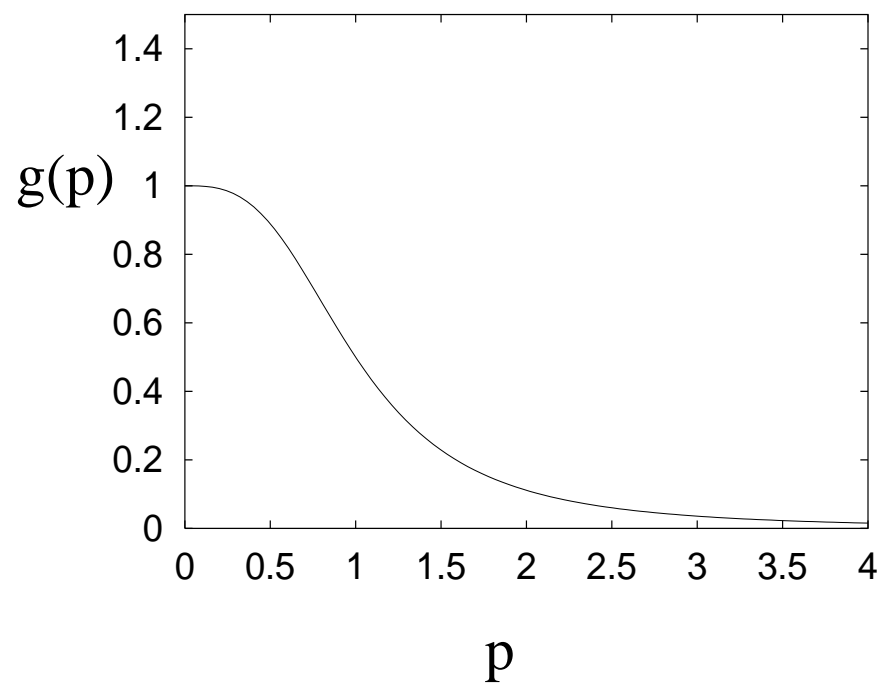

Figure 2: The Hill function $g(p)=1 /\left(1+p^{n}\right)$ with $n=3$, an example of the nonlinear (sigmoidal) feedback term used to model baroreceptor control of heart rate.

The dependence of peripheral resistance $R_{c}$ on sympathetic tone is modelled as a simple delayed linear dependence, measured by the parameter $\alpha$. $R_{c}$ varies from the minimum value $R_{c}^{0}$ to the maximum value $R_{c}^{0}(1+\alpha)$, as the sympathetic tone varies. Approximate values of the parameters are given in Table (1), and are based on those presented by Ursino [38].

Following Ottesen [29], we take $T_{s}(t)=g\left(p_{a}(t-\tau)\right) \equiv g\left(p_{a}^{\tau}\right)$, where $g$ has the sigmoidal form sketched in Fig. 2, but is otherwise quite general, and $\tau$ is the sympathetic time delay, typically $3-4$ seconds. For the parasympathetic control, we use the form $T_{p}(t)=1-g\left(p_{a}(t)\right) \equiv 1-g\left(p_{a}\right)$, which has no delay, since we are neglecting the much smaller time delays in this system.

\subsection{Rescaled Model}

We nondimensionalise our three model equations, and calculate the sizes of the resulting non-dimensional parameters. Parameter definitions and approximate values are listed in Table 2.

We let

$$
p_{a}=P_{0} p_{a}^{*}, \quad p_{v}=\rho \mu P_{0} p_{v}^{*}, \quad H=H_{0} h^{*}, \quad t=\tau t^{*} .
$$




\begin{tabular}{|l|l|l|}
\hline parameter & definition & value \\
\hline \hline$C_{a}$ & arterial compliance & $1.55 \mathrm{ml} \mathrm{mm} \mathrm{Hg}^{-1}$ \\
\hline$C_{v}$ & venous compliance & $50 \mathrm{ml} \mathrm{mm} \mathrm{Hg}^{-1}$ \\
\hline$R_{c}^{0}$ & min arteriole resistance & $0.6 \mathrm{~mm} \mathrm{Hg} \mathrm{ml}^{-1}$ \\
\hline$R_{v}$ & venous resistance & $0.016 \mathrm{~mm} \mathrm{Hg} \mathrm{s} \mathrm{ml}$ \\
\hline$\Delta V$ & stroke volume & $50 \mathrm{ml}$ \\
\hline$H_{0}$ & uncontrolled heart rate & $100 \mathrm{~min}^{-1}$ \\
\hline$P_{0}$ & arterial pressure & $100 \mathrm{~mm} \mathrm{Hg}$ \\
\hline$\tau$ & sympathetic delay & $3 \mathrm{~s}$ \\
\hline$V_{H}$ & vagal tone & $1.17 \mathrm{~s}^{-2}$ \\
\hline$\beta_{H}$ & sympathetic control of heart rate & $0.84 \mathrm{~s}^{-2}$ \\
\hline$\alpha$ & sympathetic effect on $R_{c}$ & 1.3 \\
\hline$\gamma$ & vagal damping of $\beta_{H}$ & 0.2 \\
\hline$\delta_{H}$ & relaxation time & $1.7 \mathrm{~s}^{-1}$ \\
\hline
\end{tabular}

Table 1: Parameter values

Then, immediately dropping the asterisks, the rescaled equations are

$$
\begin{aligned}
\varepsilon_{a} \dot{p_{a}} & =\frac{-p_{a}+\mu \rho p_{v}}{1+\alpha T_{s}}+\mu h, \\
\varepsilon_{v} \dot{p_{v}} & =\frac{p_{a}-\mu \rho p_{v}}{1+\alpha T_{s}}-\mu p_{v}, \\
\varepsilon_{H} \dot{h} & =\frac{\beta T_{s}}{1+\gamma T_{p}}-\nu T_{p}+\delta(1-h) .
\end{aligned}
$$

In the absence of other information, $\delta$ is taken to be one, assuming that if central nervous control was removed, the heart would return to its uncontrolled rate in times of the same order as the uncontrolled period, which is the most natural assumption.

\subsection{Model Reductions}

Since $\rho$ is small compared with the other parameters, we neglect the venous pressure $p_{v}$ in equation (6). This term does not appear in the heart rate equation (8). We rename $p_{a}$ as simply $p$, so that our system is reduced to the two equations

$$
\begin{aligned}
\varepsilon_{H} \dot{h} & =\frac{\beta g\left(p_{1}\right)}{1+\gamma[1-g(p)]}-\nu[1-g(p)]+\delta(1-h), \\
\varepsilon_{a} \dot{p} & =-\frac{p}{1+\alpha g\left(p_{1}\right)}+\mu h,
\end{aligned}
$$




\begin{tabular}{|l|l|l|}
\hline parameter & definition & value \\
\hline \hline$\varepsilon_{a}$ & $C_{a} R_{c}^{0} / \tau$ & 0.3 \\
\hline$\varepsilon_{v}$ & $C_{v} R_{c}^{0} \mu \rho / \tau$ & 0.15 \\
\hline$\varepsilon_{H}$ & $\left.1 / H_{0} \tau\right)$ & 0.18 \\
\hline$\alpha$ & sympathetic effect on $R_{c}$ & 1.3 \\
\hline$\beta$ & $\beta_{H} / H_{0}^{2}$ & 0.3 \\
\hline$\gamma$ & vagal damping of $\beta_{H}$ & 0.2 \\
\hline$\delta$ & $\delta_{H} / H_{0}$ & 1 \\
\hline$\mu$ & $R_{c}^{0} H_{0} \Delta V / P_{0}$ & 0.5 \\
\hline$\nu$ & $V_{H} / H_{0}^{2}$ & 0.4 \\
\hline$\rho$ & $R_{v} / R_{c}^{0}$ & 0.03 \\
\hline
\end{tabular}

Table 2: Nondimensional parameter definitions and values

where $p_{1} \equiv p(t-1)$ and $p \equiv p(t)$. Further simplification is possible because the time scale of response of both $h$ and $p$ is quite small. Noting that $\varepsilon_{H}$ is smaller than $\varepsilon_{a}$, we will put $\varepsilon_{H}=0$. This corresponds to considering a steady heart rate, ignoring the faster transients in the heart rate equation. However, $\varepsilon_{H}$ is not very much smaller than (is only half the size of) $\varepsilon_{a}$. Hence we check this simplification by later conducting a numerical comparison of solutions to the coupled equations (9) and (10) with the stability results from the simplified system, in figs (4) and (5). We find that the results are reassuringly close.

Then to leading order equation (9) gives

$$
h \approx 1+\frac{1}{\delta}\left[\frac{\beta g\left(p_{1}\right)}{1+\gamma[1-g(p)]}-\nu[1-g(p)]\right],
$$

and our model further reduces to the single delay-recruitment type equation

$$
\varepsilon_{a} \dot{p}=-\frac{p}{1+\alpha g\left(p_{1}\right)}+\mu\left\{1-\frac{\nu}{\delta}[1-g(p)]\right\}+\frac{\mu \beta g\left(p_{1}\right)}{\delta[1+\gamma(1-g(p))]} .
$$

\section{Analysis}

If the right-hand side of the delay-recruitment equation (12) is set equal to zero, it implicitly defines a map from $p_{1}$ to $p$. The properties of this map appear to be closely linked to the behaviour of solutions to the full delay equation, particularly for small $\varepsilon_{a}[9,13,39]$. In our case (for physiologically reasonable parameter values) the map is monotonically decreasing, so that 
it may have a two-cycle and a fixed point, but no other periodic or chaotic behaviour. It is not surprising then that our delay-recruitment equation (12) correspondingly has a steady-state and a periodic solution, and does not exhibit period-doubling or chaos.

Steady states $p_{s}$ of equation (12) satisfy

$$
\frac{p_{s}}{1+\alpha g\left(p_{s}\right)}+\frac{\mu \nu}{\delta}\left[1-g\left(p_{s}\right)\right]-\mu=\frac{\mu \beta g\left(p_{s}\right)}{\delta\left[1+\gamma\left(1-g\left(p_{s}\right)\right)\right]} .
$$

Considering the shape of $g$ in Fig. 2, and the fact that the right-hand side of equation (13) is a decreasing function of $p_{s}$ (decreasing from a value $\mu \beta / \delta \approx 0.15$ to zero as $p \rightarrow \infty)$, while the left-hand side is an increasing function (from near $-\mu$ to a positive value), it is clear that there is a unique steady state solution for a range of parameter values near our values. We now consider the stability of this steady solution.

\subsection{Stability}

Expanding $p=p_{s}+P$ in equation (12) for small disturbances $P$ about equilibrium gives

$$
\dot{P}=-B P-G P_{1}
$$

where

$$
\varepsilon_{a} B \equiv \frac{1}{1+\alpha g}+\frac{\mu \nu\left|g^{\prime}\right|}{\delta}+\frac{\mu \beta \gamma g\left|g^{\prime}\right|}{\delta[1+\gamma(1-g)]^{2}}>0
$$

and

$$
\varepsilon_{a} G \equiv \frac{p_{s} \alpha\left|g^{\prime}\right|}{(1+\alpha g)^{2}}+\frac{\mu \beta\left|g^{\prime}\right|}{\delta[1+\gamma(1-g)]}>0
$$

with $g, g^{\prime}$ being evaluated at $p_{s}$. By $P$ is meant $P(t)$, and $P_{1}$ means the delayed $P(t-1)$.

Putting $P=e^{\sigma t}$ then gives

$$
\sigma=-B-G e^{-\sigma}
$$

and instability occurs if $\operatorname{Re} \sigma>0$; the instability is oscillatory if $\operatorname{Im} \sigma \neq 0$.

This is an equation whose properties are well understood, see for example Diekmann et al. [12] or Murray [28]. It is a transcendental equation, which has an infinite number of complex roots, no more than two of which are real. The roots accumulate at the essential singularity at $\sigma=\infty$, and the set of $\operatorname{Re} \sigma$ is easily shown to be bounded above. There is an instability criterion which determines when all the roots $\sigma$ have negative real part, and this is indicated in Fig. (3). 


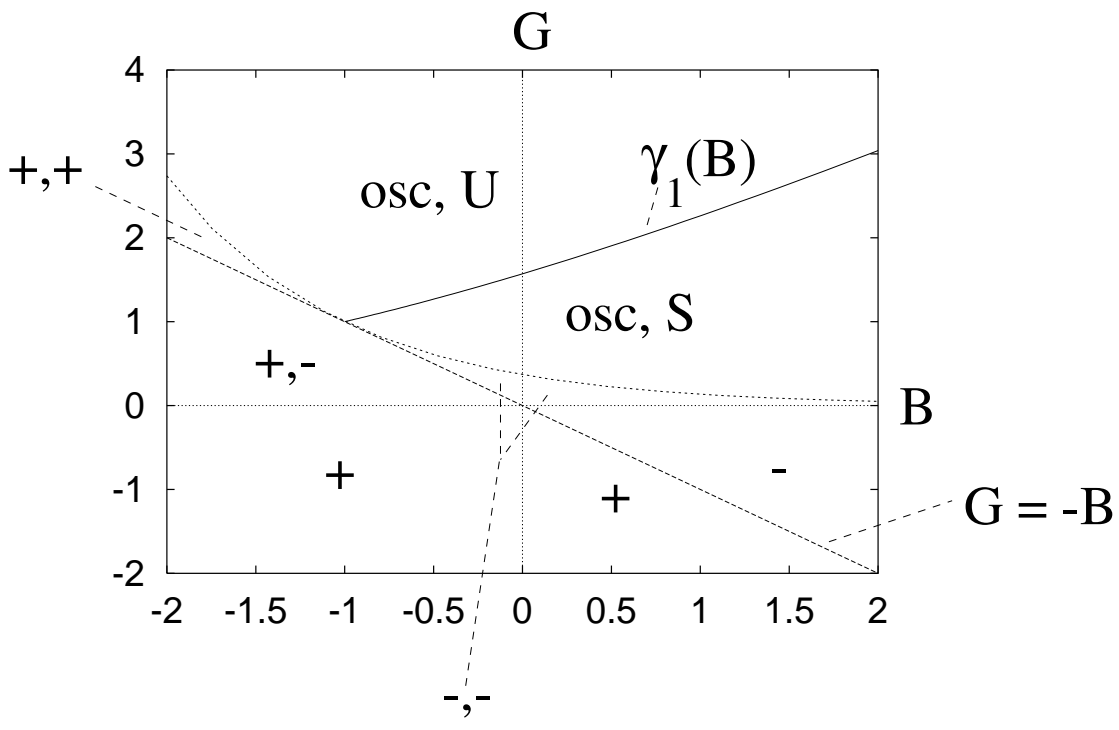

Figure 3: Stability map for the solutions of equation (17). The curves demarcate the behaviours of the two roots involved in the transition to instability. osc means oscillation and complex conjugate roots, $U$ means unstable, $S$ means stable, and + and - indicate the signs of the two roots when they are real. If only one sign is indicated, the other root has disappeared by tending to infinity. The diagram indicates that stability occurs only if $G$ lies within the sector bounded by and to the right of the two curves $G=-B$ and $G=\gamma_{1}(B)$. Note that the region of interest for this work is just the first quadrant. 
Specifically, the three curves in the figure are given by $G=-B, G=$ $\exp [-(1+B)]$, and the Hopf bifurcation curve $\gamma_{1}(B)$, which is given parametrically by

$$
B=-\frac{\Omega}{\tan \Omega}, \quad G=\frac{\Omega}{\sin \Omega},
$$

where $\Omega \in(0, \pi)$. In the present case, $B>0$ and $G>0$, so that $\Omega \in$ $(\pi / 2, \pi)$, and instability occurs if and only if $G>\gamma_{1}(B)$. The period of the resulting oscillation is $2 \pi / \Omega$, and we expect that this will be comparable to the frequency of oscillations that occur in practice. The dimensionless period $P$ of the bifurcating periodic solution is thus $P \rightarrow 2$ as $B \rightarrow \infty$, $P \rightarrow 4$ as $B \rightarrow 0$, the behaviour being monotonic along the bifurcation curve. The dimensional period is simply $P \tau$. This is consistent with 10 second Mayer waves, if the delay $\tau=3$ seconds (since the corresponding value of $B=3.3)$.

\section{Cases}

\subsection{Peripheral resistance effects}

The full stability map depends in a complicated way on the control parameters $\alpha, \beta, \gamma, \mu$ and $\nu$. In the search for simple criteria, it useful to consider the rôles of the three controls separately. We begin by considering the $\alpha-$ sympathetic control only, by taking $\alpha \neq 0$ and putting $\beta=\gamma=\nu=0$. This corresponds to investigating only the sympathetic control of peripheral resistance. From this we have that the steady state is given by

$$
h=1, \quad \mu=\frac{p}{1+\alpha g},
$$

and the parameters $B$ and $G$ are given by

$$
\varepsilon_{a} B=\frac{1}{1+\alpha g}, \quad \varepsilon_{a} G=\frac{p \alpha\left|g^{\prime}\right|}{(1+\alpha g)^{2}} .
$$

Fig. (4) shows the resultant Hopf stability curve in $\alpha-\varepsilon_{a}$ space. Oscillations occur in this simplified system when $\varepsilon_{a}$ lies below the stability curve. The figure also shows the results of direct numerical simulations of the full equations (9) and (10), and indicates that the simple system (with $\varepsilon_{H}=0$ ) gives a good approximation to the more complicated one. 


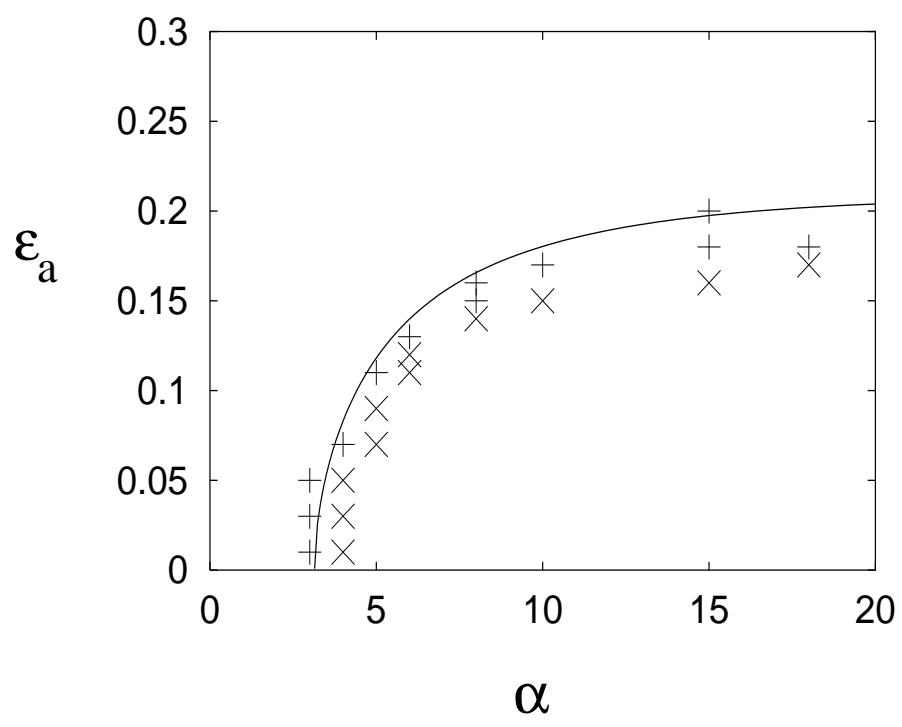

Figure 4: Stability map for $\alpha$-only control. The solid curve shows the theoretical stability limit based on the limit $\varepsilon_{H} \rightarrow 0$, together with $\beta=\gamma=$ $\nu=0$. The inclined crosses $(\times)$ indicate non-decaying oscillatory numerical solutions of the full two-dimensional system with $\varepsilon_{H}=0.18, \beta=0.3, \gamma=$ $0.2, \delta=1, \nu=0.4, \mu=0.5, n=3$. The upright crosses $(+)$ indicate where numerical results exhibit oscillatory decay to a stable steady state. It can be seen that the simple analytic asymptotic theory gives a good (over) estimate of the actual stability boundary. 


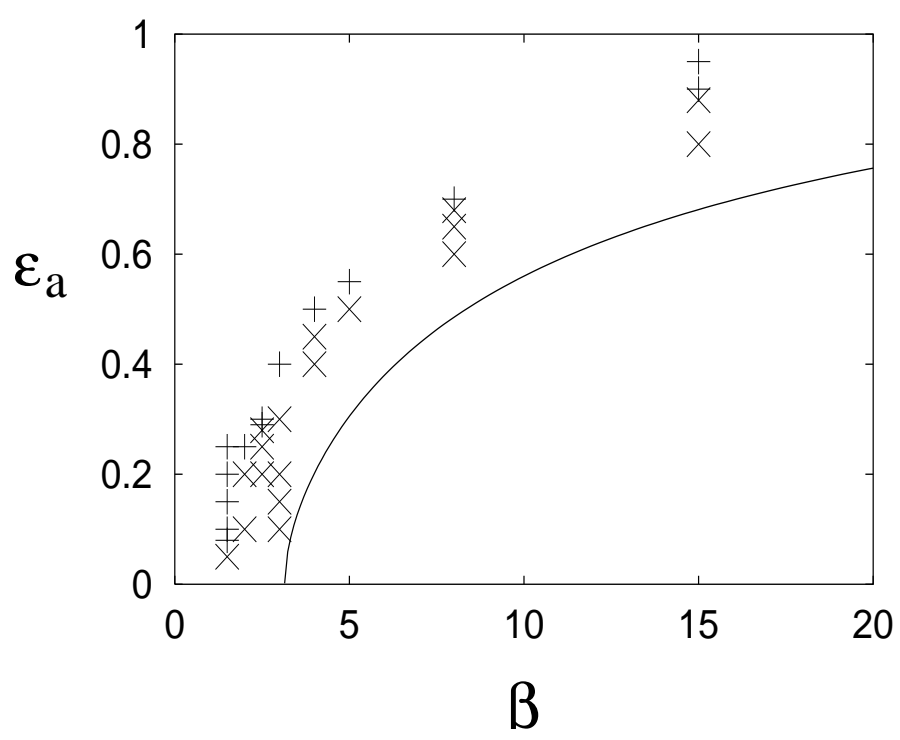

Figure 5: Stability map for $\beta$-only control. The solid curve shows the theoretical stability limit based on the limit $\varepsilon_{H} \rightarrow 0$, together with $\alpha=\gamma=$ $\nu=0$. The inclined crosses $(\times)$ indicate non-decaying oscillatory numerical solutions of the full two-dimensional system with $\varepsilon_{H}=0.18, \alpha=1.3, \gamma=$ $0.2, \delta=1, \nu=0.4, \mu=0.5, n=3$. The upright crosses ( + ) indicate where numerical results exhibit oscillatory decay to a stable steady state. It can be seen that the simple asymptotic theory gives a good (under) estimate of the actual stability boundary. 


\subsection{Heart rate effects}

The second simplified case we study is when $\beta \neq 0$, but $\alpha=\gamma=\nu=0$. This corresponds to having only sympathetic control of heart rate. In this case the steady state is

$$
h=1+\frac{\beta g}{\delta}, \quad \mu=\frac{p}{h},
$$

and the parameters $B$ and $G$ are given by

$$
\varepsilon_{a} B=1, \quad \varepsilon_{a} G=\frac{\mu \beta\left|g^{\prime}\right|}{\delta} .
$$

Fig. (5) shows the resultant Hopf stability curve in $\beta-\varepsilon_{a}$ space. Oscillations occur when $\varepsilon_{a}$ lies below the stability curve. Direct simulations of the full equations (9) and (10) again indicate that the simple system gives a good approximation to the more complicated one.

\subsection{Solely vagal effects}

Finally we consider the case where the sympathetic system is switched off, so that $\alpha=\beta=0$. In this case the equations (9) and (10) reduce to two ordinary differential equations. When these are linearised about the unique steady state, one finds that the trace of the linearised stability matrix is negative, and its determinant is positive. Thus the steady state is always stable in this case. This agrees with the results of Abbiw-Jackson and Langford [1], who also find that vagal control of heart rate does not induce instability.

\subsection{Comparison of gain versus delay}

As noted in the introduction, the cause of Mayer waves continues to be the subject of research, with disagreement over the importance of delay versus feedback gain $[1,6,34]$, and questions about the relative importance of feedback to the heart versus feedback to the peripheral resistance [23, 26, 31].

We assess the relative importance of these factors by considering their proximity to the Hopf bifurcation curve in the stability diagrams of figures (4) and (5). When parameter values are far from the curve, then the solutions of the model are strongly damped or strongly oscillatory. It is only near the curve that a change in parameter values will cause a change of behaviour. In particular, when $\alpha$ or $\beta$ are small and the associated Hopf curve is nearly vertical, the model is more sensitive to variations in $\alpha$ and $\beta$. 
For larger values of $\alpha$ and $\beta$, where the Hopf curve is flatter, the behaviour is more sensitive to variations in $\varepsilon_{a}$.

Our model allows us then to compare the relative importance for the onset of Mayer waves, of

1. the gain $\alpha$ in peripheral resistance control,

2. the gain $\beta$ in sympathetic control of heart rate, and

3. the delay $\tau$ in these controls, which appears in $\varepsilon_{a}$.

Noting that typical values are $\alpha=1.3, \beta=0.3$, and $\varepsilon_{a}=0.3$, we draw from Figs (4) and (5) the following points:

For small $\varepsilon_{a}$ (large delays), the system is not very sensitive to $\varepsilon_{a}$ (and hence to the delay $\tau$ ), compared with gain. For larger $\varepsilon_{a}$ (small delays), where the stability curves are nearly horizontal, the system is much more sensitive to delay.

The effects of heart rate control are greater than those of peripheral resistance control at values of $\varepsilon_{a} \approx 0.3$, since a $\beta$ value near $3-5$ gives oscillation, whereas no $\alpha$ values in the range zero to twenty can cause oscillation. The graphs also reveal that on average, tracking the Hopf bifurcation curve, a change in $\varepsilon_{a}$ of the order of one (with changes $\tau$ of order of a factor of 3 ) is equivalent to a change of order of a factor of fifty in $\alpha$ and of order ten in $\beta$. Hence the system is generally more sensitive to changes in time delay than to changes in feedback gain, and is more sensitive to changes in the sympathetic feedback to the heart than to peripheral resistance. This result is at odds with the ideas presented in [23, 26, 31], and needs further study.

\subsection{Ageing}

As humans age, generally their reflexes get slower, and hence we expect somewhat longer delay times for the sympathetic system. As pointed out elsewhere [1], in other models and in ours, regions of instability correspond to longer delay times. This is at first glance not consistent with clinical observations, which indicate that older people are less likely to exhibit Mayer waves [20, 22].

However, perhaps more significantly, ageing also brings impaired function of the smooth muscle walls of arterioles, resulting in less responsive peripheral resistances, together with higher overall resistances and smaller arterial compliances [35]. In terms of our model then, we would simulate ageing by decreasing the gains $\alpha$ and $\beta$ of feedback to peripheral resistance and the heart, increasing the capillary resistance $R_{c}^{0}$, and increasing the 
time delay $\tau$. Then we anticipate little change in $\varepsilon_{a}$, since increases in $\tau$ and decreases in $C_{a}$ are offset by a larger value for $R_{c}^{0}$.

The implications of these changes in parameter values are, referring to Figs (4) and (5), that ageing corresponds to a movement from the operating point $\varepsilon_{a}=0.3, \beta=0.3$, and $\alpha=1.3$ in the direction of reduced $\alpha$ and $\beta$, which is a movement away from the region of unstable oscillations. Hence ageing corresponds to a movement towards the region of greater stability in our model, which is consistent with clinical observations of reduced variability with age.

\section{Conclusions}

We have proposed a simple model for the human cardiovascular system with baroreceptor control feedback, which is an extension of a model by Ottesen. We have reduced our model to a single delay-recruitment equation, and we have studied analytically and numerically the behaviour of solutions. Steady solutions can lose stability in a Hopf bifurcation to oscillatory solutions, consistent with Mayer waves, as delays are increased, or as feedback gain is increased, or as peripheral resistance is reduced. Chaotic dynamics are not a feature of our model.

Our model indicates that sympathetic control of heart rate is more important than sympathetic control of peripheral resistance, and that solution stability is more sensitive to delay than to gain. The consequences of ageing are considered and found to be consistent with our model, with decreased gains giving more stable behaviour.

\section{Acknowledgements}

Mark McGuinness thanks the Science Faculty University Research Fund, Victoria University of Wellington, New Zealand, for the travel support that allowed this collaboration to flourish, and the Engineering and Physical Sciences Research Council (United Kingdom) for the funding that initiated this work.

\section{References}

[1] Abbiw-Jackson, R.M., Langford, W.F.: Gain-induced oscillations in blood pressure. J. Math. Biol. 37 (1998) 203-234 
[2] Berne, R.M., and Levy, M.N., Principles of Physiology, (Mosby-Year Book, Second Edn, 1996)

[3] Bertram, D., Barres, C., Cheng, Y., Julien, C.: Norepinephrine reuptake, baroreflex dynamics, and arterial pressure variability in rats. Am. J. Physiol. Regulatory Integrative Comp. Physiol. 279(4) (2000) R1257R1267

[4] Bertram, D., Barres, C., Cuisinard, G., Julien, C.: The arterial baroreceptor reflex of the rat exhibits positive feedback properties at the frequency of Mayer waves. J. Physiol. - London 513(1) (1998) 251-261

[5] Bertram, D., Barres, C., Julien, C.: Effect of desipramine on spontaneous arterial pressure oscillations in the rat. Eur. J. Pharmacology 378(3) (1999) 265-271

[6] Burgess, D.E., Hundley, J.C., Li, S-G., Randall, D.C., Brown, D.R.: First-order differential-delay equation for the baroreflex predicts the 0.4Hz blood pressure rhythm in rats. Am. J. Physiol. 273 (1997) R1878R1884

[7] Cavalcanti, S., Ursino, M.: Dynamical modelling of sympathetic and parasympathetic interplay on the baroreceptor heart rate control. Surv. Math. Ind. 7 (1997) 221-237

[8] Cevese, A., Gulli, G., Polati, E., Gottin, L., Grasso, R.: Baroreflex and oscillation of heart period at $0.1 \mathrm{~Hz}$ studied by $\alpha$-blockade and crossspectral analysis in healthy humans. J. Physiol. 531(1) (2001) 235-244

[9] Chow, S.-N., and Mallet-Paret, J.: Singularly perturbed delaydifferential equations. In Coupled Nonlinear Oscillators, eds J. Chandra and A.C. Scott, North-Holland, 1983

[10] Cooley, R.L., Montano, N., Cogliati, C., van de Borne, P., Richenbacher, W., Oren, R., Somers, V.K.: Evidence for a central origin of the low-frequency oscillation in RR-interval variability. Circulation 98 (1998) $556-561$

[11] deBoer, R.W., Karemaker, J.M., Strackee, J.: Hemodynamic fluctuations and baroreflex sensitivity in humans: a beat-to-beat model. Am. J. Physiol. 253 (Heart Circ. Physiol. 22) (1987) 680-689 
[12] Diekmann, O., van Gils, S.A., Verduyn Lunel, S.M., Walther, H.-O.: Delay Equations: Functional, Complex and Nonlinear Analysis, Applied Mathematical Sciences 110, Springer, 1995

[13] Fowler, A.C., Mathematical Models in the Applied Sciences, Cambridge Texts in Applied Mathematics, CUP 1997

[14] Gebber, G.L., Zhong, S., Zhou, S.-Y., Barman, S.M.: Nonlinear dynamics of the frequency locking of baroreceptor and sympathetic rhythms. Am. J. Physiol. 273 (Regulatory Integrative Comp. Physiol. 42) (1997) R1932-R1945

[15] Girard, A., Meilhac, B., Mouniervehier, C., Elghozi, J.L.: Effects of beta-adrenergic-blockade on short-term variability of blood-pressure and heart rate in essential hypertension. Clinical \& Exp. Hypertension 17(Iss 1-2) (1995) 15-27

[16] Greenwood, J.P., Stoker, J.B., Mary, D.: Single-unit sympathetic discharge - quantitative assessment in human hypertensive disease. Circulation 100 (1999) 1305-1310

[17] Grodins, F.S.: Integrative cardiovascular physiology: a mathematical synthesis of cardiac and blood vessel hemodynamics. Q. Rev. Biol. 34 (1959) 93-116

[18] Guyton, A., Textbook of Medical Physiology (W.B. Saunders Co., London, 1981)

[19] Guyton, A.C., Harris, J.W.: Pressoreceptor-autonomic oscillation: a probable cause of vasomotor waves. Am. J. Physiol. 165 (1951) 158-166

[20] Kaplan, D.T., Furman, M.I., Pincus, S.M., Ryan, S.M., Lipsitz, L.A., Goldberger, A.L.: Aging and the complexity of cardiovascular dynamics. Biophys. J. 59 (1991) 945-949

[21] Korner, P.: Integrative neural cardiovascular control. Physiol. Rev. 51(2) (1971) 312-367

[22] Libsitz, L.A., Goldberger, A.L.: Loss of complexity and aging: Potential applications of fractals and chaos theory to senescence. JAMA 267 (1992) 1806-1809

[23] Liu, H-K., Guild, S-J., Ringwood, J.V., Barrett, C.J., Leonard, B.L., Nguang, S-K., Navakatikyan, M.A., Malpas, S.C.: Dynamic baroreflex 
control of blood pressure: influence of the heart vs. peripheral resistance. Am. J. Physiol. Regulatory Integrative Comp. Physiol. 283 (2002) R533R542

[24] Madwed, J.B., Albrecht, P., Mark, R.G., Cohen, R.J.: Low frequency oscillations in arterial pressure and heart rate: a simple computer model. Am. J. Physiol. 256 (1989) H1573-H1579

[25] Magosso, E., Biavati, V., Ursino, M.: Role of the baroreflex in cardiovascular instability: a modeling study. Cardiov. Eng. 1(2) (2001) 101-115

[26] Malpas, S.C.: Neural influences on cardiovascular variability: possibilities and pitfalls. Am. J. Physiol. Heart Circ. Physiol. 282 (2002) H6-H20

[27] Montano, N., Gnecchi-Ruscone, T., Porta, A., Lombardi, F., Malliani, A., Barman, S.M.: Presence of vasomotor and respiratory rhythms in the discharge of single medullary neurons involved in the regulation of cardiovascular system. J. Auton. Nerv. Syst. 57 (1996) 116-122

[28] Murray, J. D. Mathematical biology. I: an introduction (Springer-Verlag, Berlin 2002) 23-27

[29] Ottesen, J.T.: Modelling of the baroreflex-feedback mechanism with time-delay. J. Math. Biol. 36 (1997) 41-63

[30] Ottesen, J.T., Olufsen, M.S., and Larsen, J.K.: Applied Mathematical Models in Human Physiology, SIAM Monographs on Mathematical Modelling and Computation, 2004.

[31] Ringwood, J.V., Malpas, S.C.: Slow oscillations in blood pressure via a nonlinear feedback model. Am. J. Physiol. Regulatory Integrative Comp. Physiol. 280 (2001) R1105-R1115

[32] Rosenblum, M., Kurths, J.: A model of neural control of the heart rate. Physica A 215 (1995) 439-450

[33] Rowel, L.B.: Human Cardiovascular Control, Oxford University Press, 1993.

[34] Seidel, H., Herzel, H.: Bifurcations in a nonlinear model of the baroreceptor-cardiac reflex. Physica D 115 (1998) 145-160

[35] Singh, N., Prasad, S., Singer, D.R., MacAllister, R.J.: Ageing is associated with impairment of nitric oxide and prostanoid dilator pathways in the human forearm. Clin. Sci. (Lond). 102(5) (2002) 595-600 
[36] Ursino, M.: Interaction between carotid baroregulation and the pulsating heart: a mathematical model. Am. J. Physiol. 275 (Heart Circ. Physiol. 44) (1998) H1733-H1747

[37] Ursino, M., Antonucci, M., Belardinelli, E.: Role of active changes in venous capacity by the carotid baroreflex: analysis with a mathematical model. Am. J. Physiol. 267 (Heart Circ. Physiol. 36) (1994) H2531-H2546

[38] Ursino, M., Fiorenzi, A., Belardinelli, E.: The role of pressure pulsatility in the carotid baroreflex control: a computer simulation study. Comput. Biol. Med. 26(4) (1996) 297-314

[39] Wattis, J.A.D.: Bifurcations and Chaos in a Differential-Delay Equation, an MSc Dissertation in Mathematical Modelling and Numerical Analysis, University of Oxford, September 1990. 